\title{
A study of the interaction of alcohol drinking and tobacco smoking among French cases of laryngeal
} cancer

\author{
PASCAL GUÉNEL, ${ }^{1}$ JEAN-FRANÇOIS CHASTANG, ${ }^{1}$ DANIÈLE LUCE, ${ }^{1}$ \\ ANNETTE LECLERC, ${ }^{1}$ AND JACQUES BRUGE RE ${ }^{2}$ \\ From Inserm U.88, ${ }^{1} 91$ boulevard de l'Hôpital, F-75634 Paris Cedex 13, and Service de Chirurgie cervico-faciale et \\ ORL, ${ }^{2}$ Institut Curie, Paris
}

SUMMARY Laryngeal cancer represents an important cause of cancer in France, and the individual effects of alcohol and tobacco on this cancer site are well known. However the problem of the interaction between these agents is less extensively documented, and the role of the high consumptions of alcohol has not been studied frequently. A case-control analysis was undertaken to investigate the joint effect of alcohol and tobacco by comparing 197 glottic and 214 supraglottic cancer cases to 4135 controls representative of the French general population. Heavy drinkers were available from the two groups of cases, the highest alcohol category being equivalent to a consumption of more than 2 litres of wine per day. The relative risks estimated for heavy drinkers and smokers were high, and the results indicated an even stronger effect of alcohol drinking and tobacco smoking on the upper part of the laryngeal region. Additive and multiplicative models were fitted to the data. The multiplicative hypothesis was found to be the most appropriate, implying that the risks associated with alcohol and tobacco multiply when the exposures occur simultaneously. The public health implications of this result and the contribution of heavy drinkers and smokers to the frequency of upper respiratory and digestive tract cancers are discussed.

The mortality from cancer of the larynx accounts for more than $5 \%$ of the cancer mortality of men in France. ${ }^{1}$ This percentage reaches about $10 \%$ for men between 40 and 60 years of age. Laryngeal cancer is the cause of about 4000 deaths per year in France, $95 \%$ of those occuring in men.

The role of alcohol and tobacco in these cancers is well known. ${ }^{2-7}$ However the role of the joint exposure to both agents acting together is less firmly established, but in a recent review ${ }^{8}$ Saracci concluded from the analysis of the data available for this cancer site that the multiplicative hypothesis was consistent. A large data set on laryngeal cancer among French males is analysed in this paper in order to provide additional evidence for this hypothesis. Since few studies have analysed cancers of the glottis and the supraglottis separately, we have studied the interaction for these cancers subsites independently.

The frequency of heavy smokers and particularly heavy drinkers is generally much higher in the French series of cancers of the upper respiratory and digestive tract (URDT) ${ }^{4}$ than in other similar published data. The set of data used in the present investigation therefore allowed the study of interaction of alcohol and tobacco to be made for high alcohol consumptions.

\section{Material and methods}

The cases of laryngeal cancer were selected from a registry of patients with URDT cancers seen at the Head and Neck Department of the Curie Institute in Paris, one of the largest cancer treatment centres in France. The registry includes all the cases of cancer diagnosed in the Department between 1975 and 1985. Age, sex, mean daily consumption of alcohol by type of alcoholic beverage, mean daily tobacco consumption by type of tobacco smoked, site of cancer coded according to ICD-8th, and histological type were recorded, along with other socio-demographic and occupational information. A detailed description of this case registry has been made earlier. ${ }^{7}$

A sample of the French general population compiled during a national survey on Health and Medical care ${ }^{9}$ carried out in 1980 and 1981 was selected as the control group. More than 16000 
persons over 15 years of age and identified from a random sample of 7323 households, were interviewed throughout France. The results were tabulated by sex, age, levels of alcohol and tobacco consumption, and smoking habit (current or ex-smoker) at the time of the survey.

Only males over 25 years of age were included into the study groups. Case and control ex-smokers were excluded since the previous tobacco consumption of ex-smokers in the general population sample was unknown. Among cases, only squamous cell carcinomas were analysed. Using these criteria of inclusion, 4135 controls were retained and compared to 197 cases with cancer of the glottis (intrinsic larynx; ICD-8th: 161.5) and to 214 cases with cancer of the supraglottis (extrinsic larynx; ICD-8th: 161.4). A classical case-control approach was used for the analysis, which was carried out separately for each of the cancer subsites.

It should be emphasised that the data were collected during medical consultations for the cancer cases and during interviews at home for the controls. Hence it was necessary to test the validity of the comparison group with regard to the information recorded for alcohol and tobacco. This was done using a third group extracted from the case registry and interviewed in the same conditions as the laryngeal cancer cases. This group included nasal and paranasal cancers, URDT adenocarcinomas and lymphomas, that are not known to be related to alcohol and tobacco. Eighty two males were identified in this way and were compared to the general population sample. Attention was first focused on the differences that might have occurred in recording alcohol consumption. Alcoholrelated relative risks (RRs) were calculated controlling for age and tobacco. No differences between groups were observed, and among the heaviest drinkers (more than 100 grams of pure alcohol per day), the RR was exactly equal to $1 \cdot 0$. The information recorded for tobacco was compared in the same way and similar results were found.

The consumption of tobacco was converted in grams per day using the following equivalences: 1 gram of tobacco $=1$ cigarette $=1 / 2$ cigarillos $=1 / 5$ cigar. The consumption of alcohol was converted into grams of pure alcohol per day in the analysis. For cases, the ethanol intake was evaluated from the degree of the alcoholic beverage and from the total amount ingested in litres per day (eg, 1 litre of wine $10 \%=80$ grams of pure alcohol). The alcohol consumption of the controls was recorded by number of glasses per day. It is generally accepted that the amount of ethanol in one glass of any alcoholic beverage ranges from 10 to 15 grams of ethanol. The highest estimate (15 grams) was used in the present study for two reasons: (i) the alcohol consumption of the controls and of the group of cancer cases not related to alcohol and tobacco (see above) agree quite well with this estimate; and (ii) the possible underestimation of the alcohol consumption remaining among controls would be partially corrected using a somewhat high equivalence for measuring the pure alcohol content of one glass.

Age-adjusted RRs were computed using MantelHaenszel estimates. Indicator variables were used for age, alcohol, and tobacco in the models. The data were fitted to the additive model using a non-linear regression analysis. The multiplicative models were computed using a stepwise logistic analysis as described by Breslow. ${ }^{10}$ Goodness of fit statistics were used for comparison of models.

\section{Results}

Table 1 shows the distribution of the laryngeal cancer cases and of the controls by alcohol and tobacco category. None of the cancer cases was both nonsmoker and non-drinker. Hence the lowest alcohol and lowest tobacco categories, which were used as references for the computation of the RRs, include the smokers of less than 10 grams of tobacco, and the drinkers of less than $\mathbf{4 0}$ grams of ethanol per day, respectively. The age-adjusted $R R s$ associated with the combined exposures to alcohol and tobacco were computed for each cell of the cross tabulation as shown in table 2 for the cancers of the glottis, and in table 3 for the cancers of the supraglottis. Looking at every column and every row of these tables, one can observe important rises of the RRs as the alcohol and the tobacco consumption increases. It should also be noticed that the excess risk (excess risk $=\mathbf{R} R-1$ ) observed for the combined exposures are generally much higher than the sum of the excess risks observed in the corresponding reference categories, suggesting a more than additive effect.

Table 1 Distribution of the cases of glottic and supraglotic cancers and of the controls according to the consumption of alcohol and tobacco

\begin{tabular}{|c|c|c|c|c|c|}
\hline \multirow[t]{2}{*}{ Tobacco (g/day) } & \multicolumn{5}{|c|}{ Alcohol (g/day) } \\
\hline & & $0-39$ & $40-99$ & $100-159$ & $160+$ \\
\hline \multirow{3}{*}{$0-9$} & glottis & 9 & 10 & 2 & 1 \\
\hline & supraglottis & 1 & 2 & 1 & 2 \\
\hline & controls & 1211 & 769 & 116 & 27 \\
\hline \multirow{3}{*}{$10-19$} & glottis & 1 & 8 & 8 & 5 \\
\hline & supraglottis & 1 & 9 & 6 & 5 \\
\hline & controls & 340 & 349 & 76 & 20 \\
\hline & glottis & 13 & 23 & 15 & 18 \\
\hline \multirow[t]{2}{*}{$20-29$} & supraglottis & 9 & 18 & 25 & 28 \\
\hline & controls & 352 & 350 & 109 & 30 \\
\hline \multirow{3}{*}{$30+$} & glottis & 12 & 21 & 14 & 37 \\
\hline & supraglottis & 6 & 21 & 32 & 48 \\
\hline & controls & 145 & 157 & 58 & 26 \\
\hline
\end{tabular}


Table 2 Age-adjusted relative risks ${ }^{*}$ associated with the combined categories of alcohol and tobacco consumption in cancer of the glottis. The $95 \%$ confidence interval is indicated in parentheses.

\begin{tabular}{lllll}
\hline \multirow{2}{*}{ Tobacco (g/day) } & \multicolumn{4}{l}{ Alcohol ( $\mathrm{g} /$ day) } \\
\cline { 2 - 5 } & $0-39$ & $40-99$ & $100-159$ & $160+$ \\
\hline $0-9$ & $1 \cdot 0$ & $1 \cdot 6$ & $2 \cdot 8$ & $5 \cdot 1$ \\
& & $(0 \cdot 6-4 \cdot 1)$ & $(1 \cdot 2-15 \cdot 2)$ & $(2 \cdot 3-53 \cdot 8)$ \\
$10-19$ & $0 \cdot 4$ & $2 \cdot 9$ & $15 \cdot 1$ & $40 \cdot 9$ \\
& $(0 \cdot 2-4 \cdot 5)$ & $(1 \cdot 1-8 \cdot 0)$ & $(5 \cdot 2-43 \cdot 4)$ & $(10 \cdot 3-191 \cdot 5)$ \\
$20-29$ & $9 \cdot 3$ & $12 \cdot 3$ & $26 \cdot 4$ & $125 \cdot 3$ \\
& $(4 \cdot 9-36 \cdot 4)$ & $(4 \cdot 3-27 \cdot 5)$ & $(7 \cdot 8-62 \cdot 3)$ & $(34 \cdot 1-367 \cdot 4)$ \\
$30+$ & $19 \cdot 2$ & $27 \cdot 4$ & $48 \cdot 9$ & $289 \cdot 4$ \\
\multicolumn{5}{l}{} \\
F age-adjustment on 4 age categories: less than 45; 45-54; 55-64; greater than or \\
equal to 65.
\end{tabular}

Table 3 Age-adjusted relative risks ${ }^{*}$ associated with the combined categories of alcohol and tobacco consumption in cancer of the supraglotis. The $95 \%$ confidence interval is indicated in parentheses.

\begin{tabular}{lllll}
\hline \multirow{2}{*}{ Tobacco (g/day) } & \multicolumn{4}{l}{ Alcohol $(\mathrm{g} /$ day) } \\
\cline { 2 - 5 } & $0-39$ & $40-99$ & $100-159$ & $160+$ \\
\hline $0-9$ & $1 \cdot 0$ & $2 \cdot 6$ & $7 \cdot 3$ & $50 \cdot 6$ \\
& & $(0 \cdot 3-10 \cdot 4)$ & $(1 \cdot 6-57 \cdot 3)$ & $(8 \cdot 4-280 \cdot 2)$ \\
$10-19$ & $3 \cdot 4$ & $27 \cdot 5$ & $75 \cdot 4$ & $115 \cdot 5$ \\
& $(0 \cdot 6-20 \cdot 9)$ & $(2 \cdot 1-49 \cdot 8)$ & $(8 \cdot 4-187 \cdot 0)$ & $(22 \cdot 8-671 \cdot 0)$ \\
$20-29$ & $32 \cdot 3$ & $48 \cdot 5$ & $180 \cdot 7$ & $647 \cdot 7$ \\
$30+$ & $(4 \cdot 4-82 \cdot 1)$ & $(6 \cdot 7-101 \cdot 0)$ & $(27 \cdot 3-415 \cdot 2)$ & $(106 \cdot 4-1749 \cdot 1)$ \\
& $46 \cdot 8$ & $132 \cdot 3$ & $530 \cdot 6$ & $1094 \cdot 2$ \\
& $(6 \cdot 7-152 \cdot 6)$ & $(16 \cdot 6-283 \cdot 8)$ & $(77 \cdot 7-1175 \cdot 7)$ & $(185 \cdot 8-2970 \cdot 7)$ \\
\hline
\end{tabular}

age-adjustment on 4 age categories: less than $45 ; 45-54 ; 55-64$; greater than or equal to 65 .

Additive and multiplicative models were fitted to the data using indicator variables for alcohol, tobacco and age. Expected numbers of cases in the combined alcohol and tobacco categories were calculated under both models and compared to the observed numbers. Tables 4 and 5 show the results for the cancers of the

Table 4 Observed numbers of cancers of the glottis and expected numbers under the additive and the multiplicative models by alcohol and tobacco category.

\begin{tabular}{|c|c|c|c|c|}
\hline $\begin{array}{l}\text { Tobacco } \\
\text { (g/day) }\end{array}$ & $\begin{array}{l}\text { Alcohol } \\
\text { (g/day) }\end{array}$ & Observed & Additive & Multiplicative \\
\hline $0-9$ & $\begin{array}{l}0-39 \\
40-99 \\
100-159 \\
160+\end{array}$ & $\begin{array}{r}9 \\
10 \\
2 \\
1\end{array}$ & $\begin{array}{r}7 \cdot 5 \\
12.2 \\
6.5 \\
9 \cdot 1\end{array}$ & $\begin{array}{l}7 \cdot 9 \\
9 \cdot 2 \\
2 \cdot 5 \\
2 \cdot 7\end{array}$ \\
\hline $10-19$ & $\begin{array}{l}0-39 \\
40-99 \\
100-159 \\
160+\end{array}$ & $\begin{array}{l}1 \\
8 \\
8 \\
5\end{array}$ & $\begin{array}{l}2 \cdot 4 \\
5 \cdot 5 \\
5 \cdot 6 \\
7 \cdot 4\end{array}$ & $\begin{array}{l}4.9 \\
8.6 \\
4.7 \\
3.7\end{array}$ \\
\hline $20-29$ & $\begin{array}{l}0-39 \\
40-99 \\
100-159 \\
160+\end{array}$ & $\begin{array}{l}13 \\
23 \\
15 \\
18\end{array}$ & $\begin{array}{l}15.5 \\
22.8 \\
11.4 \\
15.8\end{array}$ & $\begin{array}{l}11 \cdot 4 \\
23 \cdot 4 \\
15 \cdot 8 \\
18 \cdot 3\end{array}$ \\
\hline $30+$ & $\begin{array}{l}0-39 \\
40-99 \\
100-159 \\
160+\end{array}$ & $\begin{array}{l}12 \\
21 \\
14 \\
37\end{array}$ & $\begin{array}{l}16 \cdot 2 \\
22 \cdot 4 \\
11 \cdot 2 \\
25 \cdot 0\end{array}$ & $\begin{array}{l}10.7 \\
20.8 \\
15.9 \\
36.5\end{array}$ \\
\hline \multicolumn{2}{|c|}{ Log of the likelihood } & & $-543 \cdot 2$ & -526.4 \\
\hline
\end{tabular}

Table 5 Observed numbers of cancers of the supraglottis and expected numbers under the additive and the multiplicative models by alcohol and tobacco category.

\begin{tabular}{|c|c|c|c|c|}
\hline $\begin{array}{l}\text { Tobacco } \\
\text { (g/day) }\end{array}$ & $\begin{array}{l}\text { Alcohol } \\
\text { (g/day) }\end{array}$ & Observed & Additive & Multiplicative \\
\hline $0-9$ & $\begin{array}{l}0-39 \\
40-99 \\
100-159 \\
160+\end{array}$ & $\begin{array}{l}1 \\
2 \\
1 \\
2\end{array}$ & $\begin{array}{r}0.8 \\
2.7 \\
8.6 \\
11.4\end{array}$ & $\begin{array}{l}1.4 \\
2.3 \\
1.2 \\
1.2\end{array}$ \\
\hline $10-19$ & $\begin{array}{l}0-39 \\
40-99 \\
100-159 \\
160+\end{array}$ & $\begin{array}{l}1 \\
9 \\
6 \\
5\end{array}$ & $\begin{array}{l}3.8 \\
5.2 \\
7.9 \\
8.7\end{array}$ & $\begin{array}{l}2.7 \\
6.9 \\
6.4 \\
5 \cdot 0\end{array}$ \\
\hline $20-29$ & $\begin{array}{l}0-39 \\
40-99 \\
100-159 \\
160+\end{array}$ & $\begin{array}{r}9 \\
18 \\
25 \\
28\end{array}$ & $\begin{array}{l}12.8 \\
18.8 \\
15.4 \\
23.3\end{array}$ & $\begin{array}{r}6.5 \\
20 \cdot 4 \\
26 \cdot 4 \\
26.7\end{array}$ \\
\hline $30+$ & $\begin{array}{l}0-39 \\
40-99 \\
100-159 \\
160+\end{array}$ & $\begin{array}{r}6 \\
21 \\
32 \\
48\end{array}$ & $\begin{array}{l}15.9 \\
24.7 \\
17.7 \\
35.3\end{array}$ & $\begin{array}{r}6.4 \\
20.5 \\
30.0 \\
50.0\end{array}$ \\
\hline \multicolumn{2}{|c|}{ Log of the likelihood } & & $-495 \cdot 2$ & $-452 \cdot 3$ \\
\hline
\end{tabular}

glottis and the supraglottis. The number of cases predicted by the additive model is generally far from the observed number. This result shows that an additive hypothesis does not fit the data adequately, while the observed and the expected numbers are close to each other under the muliplicative hypothesis.

In order to estimate a possible deviation from the multiplicative hypothesis, a logistic model with crossproduct variables alcohol $\times$ tobacco was compared to the simple multiplicative model. No statistically significant improvement of the goodness of fit was observed (the goodness of fit statistic was for glottis: $\chi^{2}=10.20$ with $9 \mathrm{df}, \mathrm{p}=0.33$; and for supraglottis:

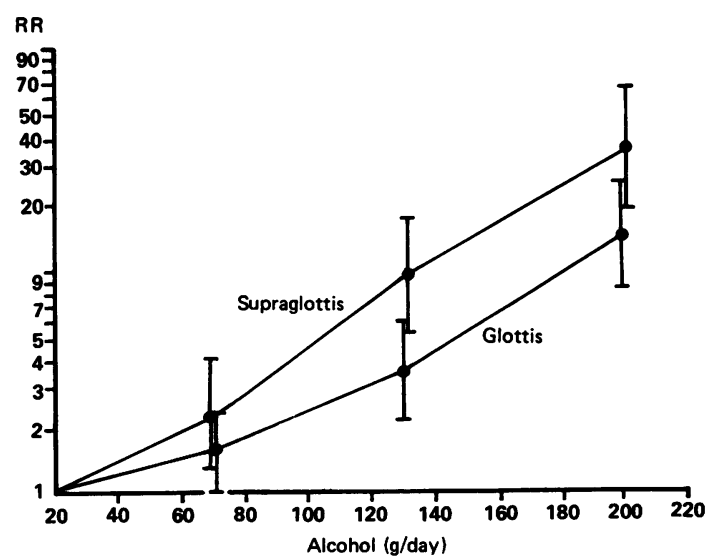

Fig 1. Relative risks associated with alcohol in cancers of the glottis and the supraglottis under the multiplicative hypothesis. RRs are adjusted by age and by tobacco consumption. The vertical bar represents the $95 \%$ confidence interval. The values of the RRs $(95 \% C I)$ are for glottis: $R R_{0 / 39 g}=1.0$; $R R_{40 / 99 g}=1.6 \quad(1.0-2.4) ; \quad R R_{100 / 159 g}=3.6 \quad(2.2-6.0)$; $R R_{160+g}=14.9(8.7-25.4)$; for supraglottis: $R R_{0 / 39 g}=1.0$; $R_{R}{ }_{40 / 99 \mathrm{~g}}=2 \cdot 3 \quad(1 \cdot 3-4 \cdot 1) ; R_{R} \quad 100 / 159 \mathrm{~g}=9 \cdot 8 \quad(5 \cdot 5-17 \cdot 6)$; $R R_{160+g}=35 \cdot 7(19 \cdot 2-66 \cdot 5)$. 
RR

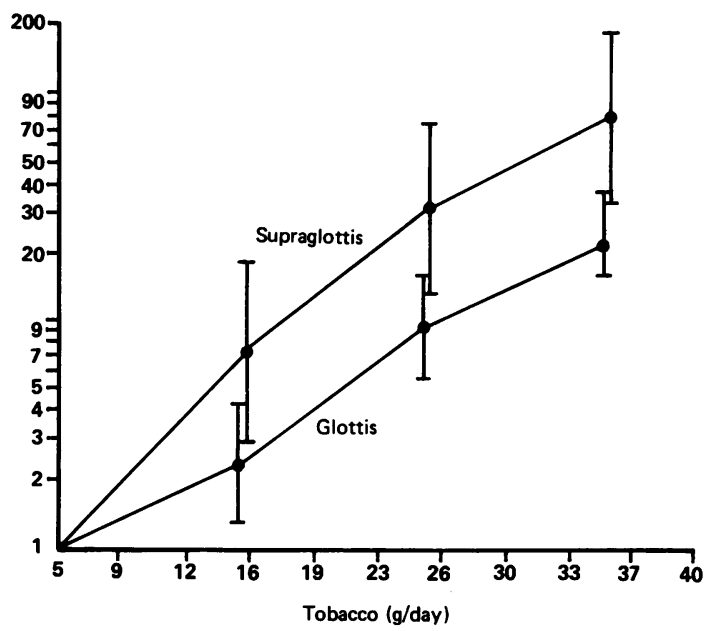

Fig 2. Relative risks associated with tobacco in cancers of the glottis and the supraglottis under the multiplicative hypothesis. $R R$ are adjusted by age and by tobacco consumption. The vertical bar represents the $95 \%$ confidence interval. The values of the $R R s(95 \% C I)$ are for glottis: $R R_{0 / 9 g}=1.0$; $R R_{10 / 19 g}=2.3 \quad(1 \cdot 3-4 \cdot 3) ; \quad R R_{20 / 29 g}=9.5 \quad(5 \cdot 6-16 \cdot 1)$; $R R_{30+g}=22 \cdot 2$ (12.9-38.3); for supraglottis: $R R_{0 / 9 g}=1 \cdot 0$; $R R_{10 / 19 g}=7.3(2.9-18.4) ; R_{20 / 29 g}=31.8$ (13.4-75.5); $R R_{30+g}=80 \cdot 5(33 \cdot 6-193 \cdot 1)$.

$\chi^{2}=4.78$ with $\left.9 \mathrm{df}, \mathrm{p}=0.85\right)$. It thus appears that the multiplicative hypothesis fits the data quite well.

The specific alcohol RRs (adjusted for age and tobacco consumption), as calculated by the multiplicative model, are presented in figure 1. A consistent dose-response relationship is observed for the two subsites. It can also be seen that the RRs for supraglottic cancer are constantly higher than those for glottic cancer. A discrepancy between cancer sites is also observed for tobacco-associated RRs (adjusted for age and alcohol consumption) shown in figure 2, the effects of tobacco on supraglottic cancer being stronger than on glottic cancer.

\section{Discussion}

The high values of RRs observed in this set of data confirm the adverse effects of alcohol and tobacco on the laryngeal region. The RRs associated with tobacco agree with the values found in other studies. ${ }^{3611}$ However heavy drinkers were not usually represented in these investigations. In the present study the effect of alcohol among heavy drinkers (the category 160 grams of ethanol or more is equivalent to 2 litres of wine or more per day) was important. Moreover the multiplicative model was found to be the most accurate to describe the combined effects of alcohol and tobacco. It is worth noting that exposure to alcohol alone seems to enhance the risk of cancer, since significant excess risks associated with alcohol are observed among moderate smokers. The RRs found in the cancers of the supraglottis were also higher than in the cancers of the glottis, suggesting a stronger effect of alcohol and tobacco smoke on the upper part of the laryngeal region.

The comparability of cases and controls poses a problem for discussion. It was noted that the interview was undertaken in different ways for both groups. This may have led to an underestimation of the alcohol and tobacco consumption in the controls and, as a consequence, to an overestimation of the RRs. Since no differences in the recorded consumptions were observed between controls and a group of URDT cancers unrelated to alcohol and tobacco, this bias was thought to be relatively weak, but may remain. Moreover the control group was drawn from a sample of the French general population over a 2-year period (1980-81), while the cases were drawn from a registry of patients living in the Paris area and diagnosed during an 11-year period (1975-1985). This did not allow us to control for potential confounding factors relating to the region or to the period of the data collection. However the consumption of alcohol and tobacco have not changed dramatically over the study period, and the habits of alcohol drinking and tobacco smoking have no special features in the Paris area. The control group had the particular advantage of providing a very large sample representative of the general population, allowing a primary analysis of tobacco/alcohol interaction in a specific group of cancer cases. More sophisticated statistical methods exploring the best fit between additive and multiplicative models ${ }^{12}$ were not used in the present study. However the simpler analysis provided here shows striking results which fit the data satisfactorily.

Previous studies of the interaction between alcohol and tobacco in cancer of the larynx generally concluded that there was more than an additive effect, ie, an effect of combined exposure to both agents which was greater than the sum of the effects due to each agent alone. ${ }^{1314}$ Saracci concluded in a recent review about interaction of other agents with tobacco smoking ${ }^{8}$ that the findings from the published studies were consistent with the multiplicative hypothesis. This conclusion is supported by our findings. The multiplicative effects of alcohol and tobacco imply that the specific contribution to the occurence of laryngeal cancer caused by the joint exposure to these agents increases in line with their increased consumption. The percent of total excess risk specifically due to this interaction was used by Saracci to describe the data analysed in his review, the term positive interaction, or synergy, indicating an excess risk over additivity. As an example, this percentage was found to be close to 90 for both cancer sites in the 
highest alcohol-tobacco category of the present data set, ie, the specific contribution of the interaction due to the combined exposures to more than 30 cigarettes and to more than 160 grams of ethanol per day would be responsible for nine cancer cases out of ten in this category. Thus the conjunction between high levels of exposure to alcohol, such as those described in this paper, and tobacco smoking in a multiplicative interaction is likely to exert a strong influence on the occurrence of the cancer of the larynx.

The problem of interaction between alcohol and tobacco is not confined to laryngeal cancers, and similar analyses for other cancer sites also related to alcohol and tobacco might be helpful in understanding the underlying mechanisms. In the cancers of the mouth and the pharynx, the data sets analysed fitted either an additive or a multiplicative model. ${ }^{15-17} \mathrm{~A}$ multiplicative relation was found for oesophageal cancers, ${ }^{18}$ but on re-analysis a more than additive but less than multiplicative relation was found to be more accurate. ${ }^{12}$ Another study combining different sites of URDT cancers concluded that both multiplicative and additive hypotheses fitted the data similarly well. ${ }^{19}$ It appears that the situation for most of the URDT cancers is not clear, but it seems reasonable to assume that the degree of interaction may vary from one site to another.

Biological hypotheses, for example that alcohol may act as a solvent of carcinogens in tobacco smoke, have been suggested, ${ }^{20}$ but it has been pointed out that the biological implications of an interaction found with statistical models cannot be derived automatically. ${ }^{21}$ Moreover the carcinogenic properties of the alcohol itself are not clear, since the type of the alcoholic beverage seems to play a role in the occurence of URDT cancers. ${ }^{22}$

\section{CONCLUSION}

The results of the present study suggest that high consumption of alcohol associated with a multiplicative model of risk with tobacco smoking is likely to explain an important part of the laryngeal cancer occurrence. It should be noted, however, that other risk factors, such as occupational exposure, may also play a role but were not taken into account in this analysis. Further investigations dealing with precise subsites of cancers of the URDT region, with appropriate control groups and controlling for the type of alcohol, would provide useful contributions to the description of the interaction and its public health consequences.

Correspondence to Dr P Guénel, Inserm U.88, 91 boulevard de l'Hôpital, F-75634 Paris Cedex 13, France.

\section{References}

${ }^{1}$ INSERM. Statistique des causes médicales de décès 1979. Paris: Les éditions INSERM, 1985.

2 Wynder EI, Bross IJ, Day E. A study of the environmental factors in cancer of the larynx. Cancer 1956; 9: 86-110.

${ }^{3}$ Wynder EI, Covey LS, Mabuchi K, Muskinski M. Environmental factors in cancer of the larynx. A second look. Cancer 1976; 38: 1591-1601.

${ }^{4}$ Schwartz D, Denoix PF, Anguera G. Recherche des localisations de cancer associees aux facteurs tabac et alcool chez l'homme. Bulletin du cancer 1957; 44: 336-61.

${ }^{5}$ Schwartz D, Flamant R, Lellouch J, Denoix PF. Results of a French survey on the role of tobacco, particularly inhalation, in different cancer sites. $J$ Natl Cancer Inst 1961; 26: 1085-108.

${ }^{6}$ Rothman KJ, Cann CI, Flanders D, Fried MP. Epidemiology of laryngeal cancer. Epidemiol Rev 1980; 2: 195-209.

${ }^{7}$ Brugère J, Guénel P, Leclerc A, Rodriguez J. Differential effects of tobacco and alcohol in cancer of the larynx, pharynx, and mouth. Cancer 1986; 57: 193-7.

${ }^{8}$ Saracci $\mathbf{R}$. The interactions of tobacco smoking and other agents in cancer etiology, Epidemiol Rev 1987; 9: 175-93.

${ }^{9}$ Lelaidier S. Quelques resultats sur les consommateurs de boissons alcooliques et de tabac en France en 1980. Enquête auprès des ménages sur la santé et les soins médicaux 1980-81. SESI Informations Rapides 1983; 20: $1-12$.

${ }^{10}$ Breslow NE, Day NE. Statistical methods in cancer research. Vol. 1 The analysis of case-control studies. Lyon: IARC publications $n^{\circ} 32,1980$.

11 Williams RR, Horn JW. Association of cancer sites with tobacco and alcohol consumption and socio-economic status of patients. Interview study from the Third National Cancer Survey. J. Natl Cancer Inst 1977; 58: 525-47.

12 Breslow NE, Storer BE. General relative risk functions for case-control studies. Am J Epidemiol 1985; 122: 149-62.

13 Flanders WD, Rothman KJ. Interaction of alcohol and tobacco in laryngeal cancer. Am J Epidemiol 1982; 115: 371-9.

14 Olsen J, Sabroe S, Fasting O. Interaction of alcohol and tobacco as risk factors in the cancer of the laryngeal region. J Epidemiol Community Health 1985; 39: 165-8.

15 Rothman KJ, Keller A. The effect of joint exposure to alcohol and tobacco on risk of cancer of the mouth and pharynx., J Chronic Dis 1972; 25: 711-6.

16 Graham S, Dayal H, Rohrer T, et al. Dentition, diet, tobacco, and alcohol in the epidemiology of oral cancer. $J$ Natl Cancer Inst 1977; 59: 1611-8.

${ }^{17}$ Olsen J, Sabroe S, Ipsen J. Effect of combined alcohol and tobacco exposure on risk of cancer of the hypopharynx. $J$ Epidemiol Community Health 1985; 39: 304-7.

18 Tuyns AJ, Pequignot G, Abbatucci JS. Oesophageal cancer and alcohol consumption; importance of type of beverage. Int J Cancer 1979; 23: 443-7.

${ }^{19}$ Elwood JM, Pearson JCG, Skippen DH, Jackson SM. Alcohol, smoking, social and occupational factors in the aetiology of cancer of the oral cavity, pharynx and larynx. Int J Cancer 1984; 34: 603-12.

${ }^{20}$ McCoy GD, Hecht SS, Wynder EL. The roles of tobacco, alcohol and diet in the aetiology of upper alimentary and respiratory tract cancer. Prev Med 1980; 9: 622-9.

${ }^{21}$ Siemiaticky J, Thomas DC. Biological models and statistical interactions: an example from multistage carcinogenesis. Int J Epidemiol 1981; 10: 383-7.

22 Leclerc A, Brugère J, Luce D, Point D, Guénel P. Type of alcoholic beverage and cancer of the upper respiratory and digestive tract. Eur J Cancer Clin Oncol 1987; 23: $529-34$. 